\title{
Effect of Annealing on Exciton Diffusion in a High Performance Small Molecule Organic Photovoltaic Material
}

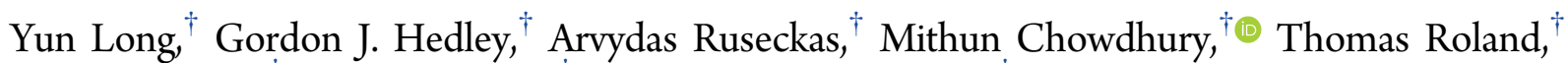 \\ Luis A Serrano, ${ }^{\ddagger}$ Graeme Cooke, ${ }^{\ddagger}$ and Ifor D. W. Samuel ${ }^{*}{ }^{\dagger}$
}

${ }^{\dagger}$ Organic Semiconductor Centre, SUPA, School of Physics and Astronomy, University of St. Andrews, North Haugh, St. Andrews, Fife KY16 9SS, United Kingdom

ॠGlasgow Centre for Physical Organic Chemistry, WESTCHEM, School of Chemistry, University of Glasgow, Glasgow G12 8QQ United Kingdom

\section{Supporting Information}

ABSTRACT: Singlet exciton diffusion was studied in the efficient organic photovoltaic electron donor material DTS$\left(\mathrm{FBTTh}_{2}\right)_{2}$. Three complementary time-resolved fluorescence measurements were performed: quenching in planar heterojunctions with an electron acceptor, exciton-exciton annihilation, and fluorescence depolarization. The average exciton diffusivity increases upon annealing from $1.6 \times 10^{-3}$ to $3.6 \times 10^{-3} \mathrm{~cm}^{2} \mathrm{~s}^{-1}$, resulting in an enhancement of the mean two-dimensional exciton diffusion length $\left(L_{D}=(4 D \tau)^{1 / 2}\right)$ from 15 to $27 \mathrm{~nm}$. About $30 \%$ of the excitons get trapped very

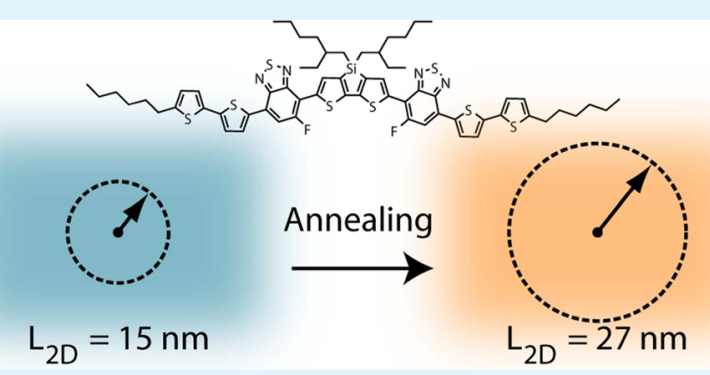
quickly in as-cast films. The high exciton diffusion coefficient of the material leads to it being able to harvest excitons efficiently from large donor domains in bulk heterojunctions.

KEYWORDS: organic solar cells, organic semiconductors, light harvesting, excitation energy transfer, bulk heterojunctions

\section{INTRODUCTION}

Organic photovoltaics (OPVs) are a promising thin-film technology for harvesting renewable solar energy to generate electricity. ${ }^{1-10}$ Key advantages of OPVs include the potential for low-cost, low-toxicity solar cells that can be produced in high volumes. Solution processability of the active layer allows for the use of cost-effective large-area deposition techniques, enabling roll-to-roll production with little material usage or waste. Solar light is harvested in OPVs through absorption of photons, leading to the formation of singlet excitons. To produce an electric current, the exciton needs to dissociate into free charge carriers. This is achieved by the use of an interface (the heterojunction) between two organic semiconductors-an electron donor and an electron acceptor. Planar heterojunctions are fabricated by sequential deposition of donor and acceptor layers while bulk heterojunctions are made by blending donor and acceptor materials. Excitons diffuse to a donor-acceptor interface where they dissociate into charge pairs. The exciton diffusion lengththe distance traveled by the exciton before it decays-is therefore a vital parameter in OPV devices, as it determines the maximum thickness of layers in planar heterojunctions and the maximum size of donor and acceptor domains in bulk heterojunctions which still give efficient charge generation. $3,5,11-13$

Bulk heterojunctions are commonly created with a conjugated polymer as an electron donor and a fullerene derivative as an electron acceptor. ${ }^{4}$ Recently, there has been a growing interest in the use of small molecules as donor materials due to their well-defined chemical structure, synthetic reproducibility, and their monodispersity. ${ }^{14-28}$ The best devices incorporating small molecules now achieve power conversion efficiencies greater than $10 \% .^{23,29}$ In the present work we have chosen to study singlet exciton diffusion in a widely studied benchmark OPV small molecule material 7,7'-(4,4-bis(2-ethylhexyl)-4H-silolo[3,2-b:4,5- $\left.b^{\prime}\right]$ dithiophene-2,6-diyl)bis (6-fluoro-4-(5'-hexyl$\left[2,2^{\prime}\right.$-bithiophen $]-5$-yl)benzo[c] $[1,2,5]$ thiadiazole)-henceforth known as DTS $\left(\text { FBTTh }_{2}\right)_{2}$. Solar cells with power conversion efficiencies of $8 \%$ have been reported using bulk heterojunctions of this molecule with the electron acceptor $\mathrm{PC}_{71}$ BM. $^{14,15,23,30,31}$

Microscopic images of DTS $\left(\mathrm{FBTTh}_{2}\right)_{2}$ blends with fullerene derivatives which were measured using atomic force microscopy (AFM) or transmission electron microscopy (TEM) showed the formation of wire-like donor domains which are more than $100 \mathrm{~nm}$ in length and $30-50 \mathrm{~nm}$ in width in blends prepared either with thermal annealing or with use of the additive 1,8-diiodooctane. ${ }^{14,27,28,32}$ A combination of neutron reflectometry and X-ray diffraction revealed a high density of small donor crystallites of $\sim 24 \mathrm{~nm}$ which are well-mixed with fullerenes in optimized blends. ${ }^{28}$ Optimization through processing dramatically improves the efficiency of devices; the power conversion

Received: January 4, 2017

Accepted: March 30, 2017

Published: March 30, 2017 
efficiency increased from $1.8 \%$ to $5.8 \%$ upon thermal annealing and to $8 \%$ when adding small amounts of 1,8 -diiodooctane and of high-molecular-weight polymer polystyrene which improves the film quality and blend morphology for charge extraction. An external quantum efficiency of up to $60 \%$ has been measured in optimized devices. ${ }^{23,30}$ Incomplete exciton harvesting can be one of the limitations to device efficiency and studies of exciton diffusion can provide useful hints on how to improve the performance.

In this paper we examine the effect of thermal annealing on the singlet exciton diffusivity and diffusion length in DTS $\left(\mathrm{FBTTh}_{2}\right)_{2}$ films using complementary experimental techniques: surface quenching of fluorescence and exciton-exciton annihilation. We determined a two-dimensional mean-squared displacement of $27 \mathrm{~nm}$ for excitons in annealed blends, which is large enough for efficient charge generation. We also studied fluorescence depolarization which indicates exciton trapping in as-cast DTS $\left(\text { FBTTh }_{2}\right)_{2}$ films.

\section{EXPERIMENTAL METHODS}

Sample Preparation. For all photoluminescence measurements the samples were made in a nitrogen atmosphere. The DTS $\left(\mathrm{FBTTh}_{2}\right)_{2}$ (powder purchased from 1-Material) was stirred overnight at $40{ }^{\circ} \mathrm{C}$ in chlorobenzene before being deposited by spin-coating. For surface quenching experiments, the samples were prepared by spin-coating a layer of DTS $\left(\mathrm{FBTTh}_{2}\right)_{2}$ on top of a cross-linked electron accepting layer of a fullerene derivative [6,6]-phenyl-C61-butyric acid styryl dendron ester (PCBSD) that acts as a fluorescence quencher. ${ }^{33,34}$ The $13 \mathrm{~nm}$ thick cross-linked PCBSD layer was deposited onto a fused silica substrate using the following process: PCBSD was dissolved overnight in dichlorobenzene solution at $75{ }^{\circ} \mathrm{C}$. The solution was then passed through a $0.1 \mu \mathrm{m}$ filter to remove undissolved residue. The filtrate was reheated to $75^{\circ} \mathrm{C}$ and spin-coated onto quartz substrates at $1500 \mathrm{rpm}$. The films spin-coated with PCBSD were then annealed at $170{ }^{\circ} \mathrm{C}$ for 40 min before being washed in chlorobenzene to remove any un-crosslinked PCBSD and then dried using a nitrogen gun. After that DTS $\left(\text { FBTTh }_{2}\right)_{2}$ was spin-coated at $1000 \mathrm{rpm}$ on top of the cross-linked PCBSD layer from solution in chlorobenzene with concentrations ranging from 2 to $6 \mathrm{mg} / \mathrm{mL}$ to form donor films of thicknesses ranging between 17 and $67 \mathrm{~nm}$, with the thicknesses being measured using variable angle spectroscopic ellipsometry (VASE). The surface roughness of the film was measured using atomic force microscopy and found to be about $0.6 \mathrm{~nm}$ (RMS). To analyze the effect of thermal annealing, one set of the films was then annealed in a nitrogen atmosphere at $130{ }^{\circ} \mathrm{C}$ for $20 \mathrm{~min}$. For exciton-exciton annihilation experiments, four DTS $\left(\text { FBTTh }_{2}\right)_{2}$ films of the same $53 \mathrm{~nm}$ thickness were spincoated from $15 \mathrm{mg} / \mathrm{mL}$ solutions at $1000 \mathrm{rpm}$ onto fused silica substrates. Three samples were then annealed on a hot plate in a nitrogen atmosphere at 70,100 , and $130{ }^{\circ} \mathrm{C}$ for $20 \mathrm{~min}$.

Fluorescence Measurements. In all fluorescence measurements, the samples were measured under active vacuum with a near normal incidence of excitation light to the plane of the film, and the emitted light was collected in the forward direction. In this geometry we preferentially excite and detect fluorescence from the molecules which have their long molecular axis orientated in the plane of the film. For surface quenching, the samples were excited using $100 \mathrm{fs}$ light pulses of $0.05 \mathrm{~nJ}$ energy at $400 \mathrm{~nm}$ and $80 \mathrm{MHz}$ repetition rate from a Spectra-Physics Mai Tai laser. Time-resolved fluorescence was measured using a Hamamatsu C6860 streak camera with a S-20 photocathode in synchroscan mode. The instrument response function of the setup was $10 \mathrm{ps}$ at full width at half-maximum (fwhm). For exciton-exciton annihilation, the samples were excited using $200 \mathrm{fs}$ laser pulses at $650 \mathrm{~nm}$ and $5 \mathrm{kHz}$ repetition rate with a pulse energy ranging from 2 to $30 \mathrm{~nJ}$ and illuminated by a beam of diameter $0.235 \mathrm{~mm}$. The pulses were generated using an optical parametric amplifier pumped by a Pharos regenerative amplifier from Light Conversion. Time-resolved fluorescence was measured using a Hamamatsu C10910-05 streak camera with a S-20ER photocathode in synchroscan mode. The instrument response function of this setup was 5 ps (fwhm). Fluorescence depolarization was measured with the same setup as exciton-exciton annihilation with a $2 \mathrm{~nJ}$ excitation pulse energy. The detection polarization was fixed with a Glan polarizer, and the excitation polarization was rotated using a half-wave plate. The timeresolved fluorescence anisotropy $r(t)$ was determined as

$$
r(t)=\frac{I_{\|}-I_{\perp}}{I_{\|}+2 I_{\perp}}
$$

where $I_{\|}$and $I_{\perp}$ are the fluorescence intensities measured with the excitation polarization set parallel and perpendicular to the detection polarization, respectively.

\section{RESULTS AND DISCUSSION}

Absorption and Fluorescence Spectra. The chemical structure, absorption, and photoluminescence (PL) spectra of DTS $\left(\mathrm{FBTTh}_{2}\right)_{2}$ solution and films are shown in Figure 1.

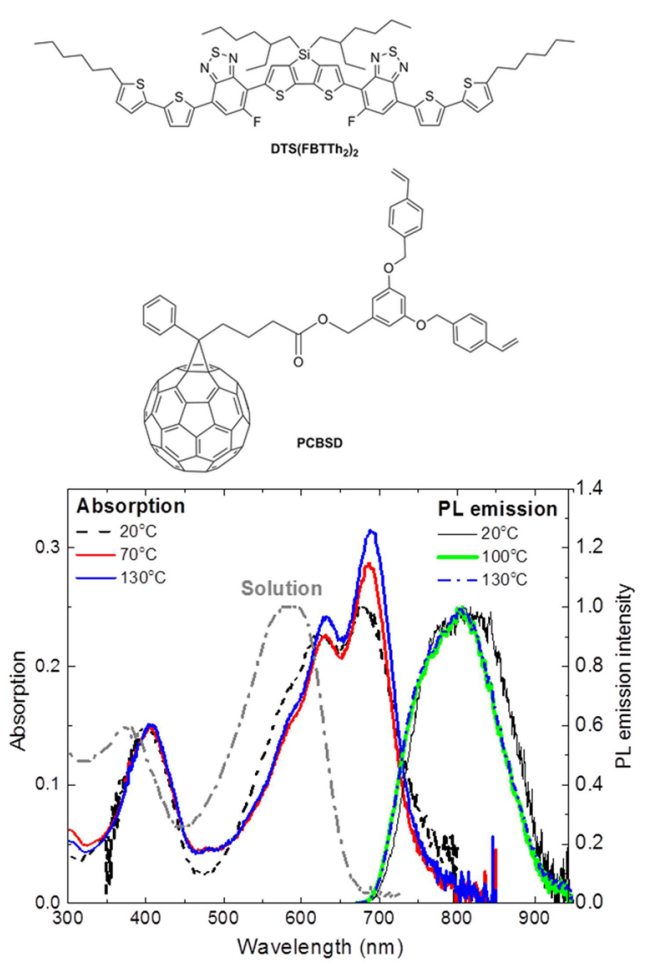

Figure 1. Chemical structures of DTS $\left(\mathrm{FBTTh}_{2}\right)_{2}$ and PCBSD and absorption and photoluminescence spectra of DTS $\left(\mathrm{FBTTh}_{2}\right)_{2}$ films spin-coated at room temperature before annealing $\left(20^{\circ} \mathrm{C}\right)$ and after annealing at 70,100 , and $130{ }^{\circ} \mathrm{C}$. The gray dash-dotted line shows absorption spectrum of this molecule in solution.

The absorption spectra of the films are substantially shifted to longer wavelength as compared to the solution spectrum and show peaks at 625 and $680 \mathrm{~nm}$ which can be attributed to vibronic $0-1$ and $0-0$ transitions, respectively. The PL peak in films is also red-shifted in comparison to that in solution as shown in Figure S1 in the Supporting Information. Similar redshift of absorption and PL spectra has been observed in a concentrated solution upon cooling and is explained by molecules aggregating and adopting a more planar conformation. ${ }^{35}$ Upon annealing the $0-0$ vibronic peak becomes stronger than the 0-1 peak, and both shift to longer wavelength which can be explained by further planarization of the molecules. The PL spectrum of the as-cast film is slightly broader and shifted to 
longer wavelength as compared to annealed films. It also shows a slight dynamic shift to longer wavelength with time, whereas the PL spectra of annealed films show no significant time evolution implying that any spectral shift occurred within our time resolution ( 2 ps, see Figure S2).

Surface Quenching of Fluorescence. Figure 2 and Figure S3 show the time-resolved PL in the films deposited
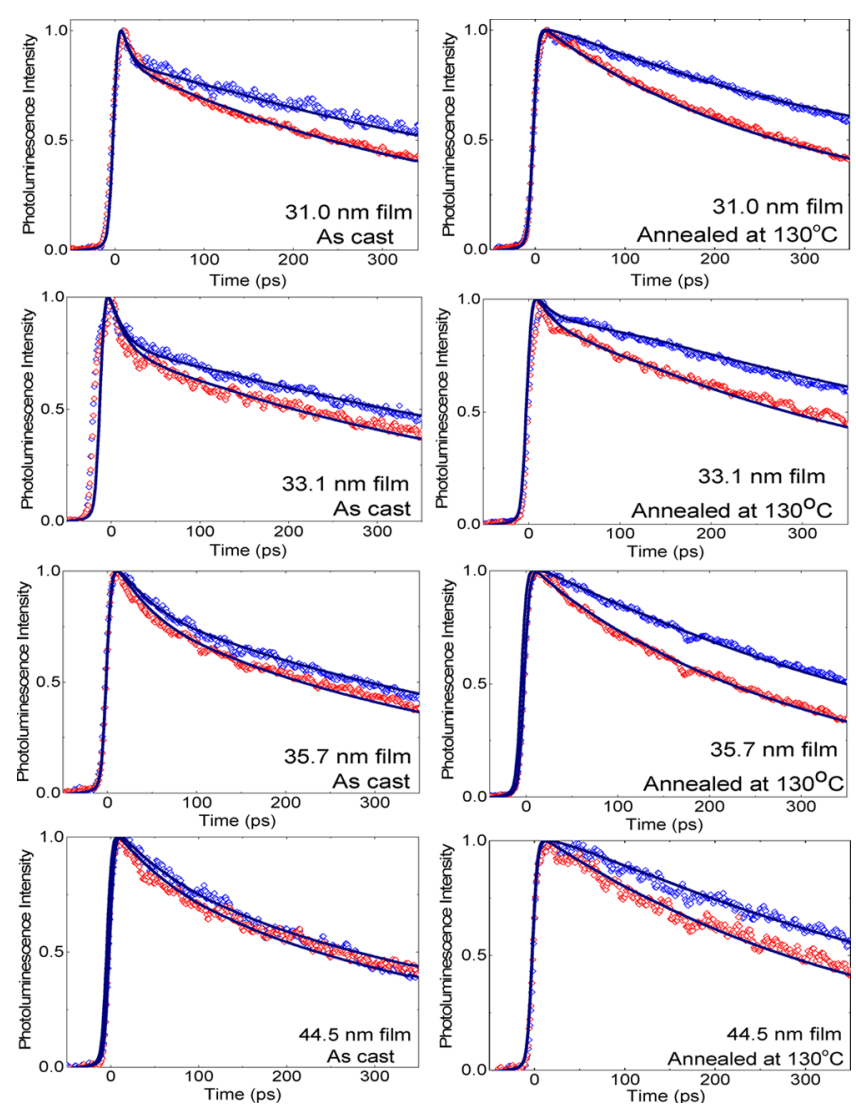

Figure 2. Time-resolved fluorescence measured in the spectral window of $750-850 \mathrm{~nm}$ in DTS $\left(\text { FBTTh }_{2}\right)_{2}$ films deposited on fused silica substrates (blue symbols) and on the cross-linked layers of fullerene PCBSD (red symbols). The left panel shows the data for as-cast films of different thicknesses while the right panel shows the data for the films of the same thickness after annealing at $130^{\circ} \mathrm{C}$. The global fits using eq 2 and boundary conditions described in the main text are shown by solid lines; they gave diffusion coefficient $D=1.6 \times 10^{-3} \mathrm{~cm}^{2} \mathrm{~s}^{-1}$ for as-cast films and $D=3.6 \times 10^{-3} \mathrm{~cm}^{2} \mathrm{~s}^{-1}$ for annealed films.

on top of a layer of cross-linked fullerene PCBSD, which quenches the fluorescence by accepting an electron from excited DTS $\left(\text { FBTTh }_{2}\right)_{2}$ in comparison with PL measured in a film of the same thickness deposited on top of nonquenching substrate of fused silica. The PL decays faster in films deposited on the fullerene layer than in the films deposited on fused silica and this difference is more pronounced in thinner films because on average excitons have to diffuse a shorter distance to the quenching interface. A higher amount of quenching is observed in the annealed films as compared to as-cast films indicating that exciton diffusion gets faster upon annealing. Because the p-DTS(FBTTh2 $)_{2}$ films were annealed at lower temperature than that used for cross-linking of the quencher layer, we expect no further change to the interface between the quencher and

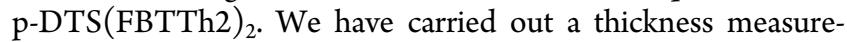
ment of the film before and after annealing using variable angle spectroscopic ellipsometry. The measured thicknesses were $39.8 \pm 0.5 \mathrm{~nm}$ for the as-cast film and $40.6 \pm 0.5 \mathrm{~nm}$ after annealing. The fluorescence intensity at the distance $x$ from the quencher layer and time $t$ after excitation is proportional to the exciton population $N(x, t)$. In order to find the diffusivity of excitons in the DTS $\left(\mathrm{FBTTh}_{2}\right)_{2}$, the dynamics of $N(x, t)$ is modeled using the one-dimensional diffusion equation ${ }^{36-38}$

$$
\frac{\partial N}{\partial t}=G+D \frac{\partial^{2} N}{\partial x^{2}}-k N-k_{\mathrm{FRET}} N
$$

In this equation, $G$ is the exciton generation rate, $D$ is the exciton diffusivity, $k$ is the rate constant of exciton decay in the bulk of the film, and $k_{\mathrm{FRET}}$ is the rate of quenching due to Förster resonance energy transfer (FRET) to the quencher. In our case the excitation pulse is very short (100 fs); hence, we assume instantaneous generation, and $k_{\mathrm{FRET}}=0$ because the PL of the film occurs at a longer wavelength than the absorption by the fullerene with no spectral overlap between them. To describe exciton quenching by electron transfer to the fullerene, we apply a boundary condition of $N=0$ at $x=0$, which represents the interface between the small molecule and the quencher. In addition, it is assumed that excitons are reflected from the top surface of the active layer, situated at $x=d$, where $d$ is the thickness of the DTS(FBTTh $)_{2}$ film. This gives a second boundary condition

$$
\left.\frac{\partial N}{\partial x}\right|_{x=d}=0
$$

The decay rate in the reference films deposited on fused silica is different for different film thicknesses, which is believed to be the result of weak quenching at the vacuum interface between the vacuum and the film. ${ }^{38,39}$ Therefore, in our data analysis we fitted the reference decay for each thickness with a three-exponential decay function and then solved eq 1 numerically with boundary conditions to find the additional decay which occurs because of exciton diffusion to the quencher. For each distinct film thickness, the exciton population $N(x, t)$ was integrated over $x$ (across the film) and convoluted with the instrument response function to generate PL decay curves for variable diffusivity $D$. The fit to PL decays was performed globally across all thicknesses with the diffusivity as the only fitting parameter. We obtain a diffusivity of $D=(1.6 \pm 0.3) \times 10^{-3} \mathrm{~cm}^{2} \mathrm{~s}^{-1}$ for the as-cast films and $D=(3.6 \pm 0.7) \times 10^{-3} \mathrm{~cm}^{2} \mathrm{~s}^{-1}$ for the annealed films. This indicates that excitons diffuse faster to the planar heterojunction with the fullerene derivative in annealed films. As annealing could conceivably change the orientation of molecules at the interface with the fullerene, we have also used excitonexciton annihilation to study exciton diffusion. Exciton-exciton annihilation is a bulk measurement which does not depend on the orientation of the molecules with respect to the substrate.

Exciton-Exciton Annihilation. The excitons diffuse within the material and annihilate when they encounter each other, causing a faster decay of fluorescence. One exciton decays by energy transfer to the other, to give a single higher energy exciton. This exciton then very quickly relaxes back to the lowest excited state, and as a result one exciton is lost per encounter. Figure 3 shows the fluorescence decays measured in as-cast films and films annealed at temperatures of 70,100 , and $130{ }^{\circ} \mathrm{C}$, using both the oscillator powered laser with pulses of lower energy and using a wide range of excitation pulse energies $E_{\mathrm{p}}$ generated by the amplified laser. The initial population of excitons is 

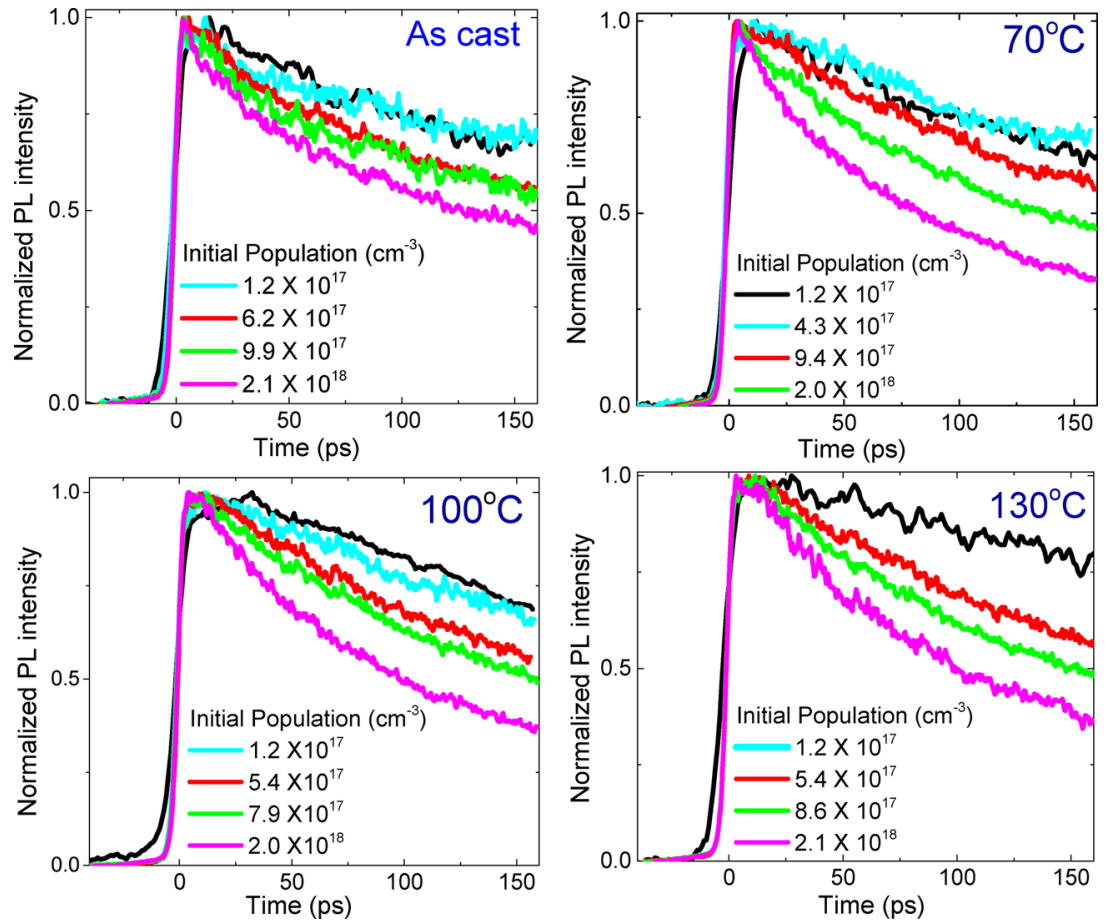

Figure 3. Time-resolved photoluminescence measured in a $750-850 \mathrm{~nm}$ window in as-cast film and films annealed at different temperatures for different initial exciton populations. The black line shows the PL kinetics measured with very low initial exciton population of $\sim 10^{15} \mathrm{~cm}^{-3}$.

determined by a number of absorbed photons per illuminated volume of the film using

$$
N(0)=\frac{E_{\mathrm{p}}}{V} \frac{\lambda}{h c}\left(1-10^{-A}\right)
$$

Here $V$ is the volume of the film illuminated by the excitation beam, $\lambda$ is the excitation wavelength, $h$ is Planck's constant, $c$ is the speed of light in air, and $A$ is the absorbance of the film at the excitation wavelength. Figure 3 shows that the fluorescence decays became faster at higher excitation densities as expected due to exciton-exciton annihilation.

The fluorescence intensity at time $t$ is proportional to the exciton population $N(t)$ which can be described by the rate equation:

$$
\frac{\mathrm{d} N}{\mathrm{~d} t}=G-k N-\gamma N^{2}
$$

Here $G$ is the exciton generation rate, $k$ is the rate constant of exciton decay measured at low initial exciton population where exciton-exciton encounter probability is very low $\left(<10^{15} \mathrm{~cm}^{-3}\right.$ in our case), and $\gamma$ is the annihilation rate constant. For instantaneous exciton generation and time-independent $\gamma$ the reciprocal of the analytical solution to eq 5 can be written as

$$
\frac{1}{N(t)}=\left(\frac{1}{N(0)}+\frac{\gamma}{k}\right) \exp (k t)-\frac{\gamma}{k}
$$

The rate $k=1 / \tau$ was obtained from the PL decays in $53 \mathrm{~nm}$ thick films measured at low excitation intensities of approximately $\sim 10^{15} \mathrm{~cm}^{-3}$ which showed single-exponential decays with the time constant $\tau$ (shown in Figure S4). In Figure 4 the reciprocal of the exciton population is plotted as a function of $\exp (k t)$, which should show a linear dependence for the time independent $\gamma$ according to eq 6 . The global linear fits to this dependence gave a single value of the annihilation rate $\gamma$ for each film. It was observed that all annealed films show similar $\gamma$ values, which were almost twice the value obtained in the as-cast film, suggesting that annealing doubles exciton diffusivity. The fact that our data can be described well using a time-independent annihilation rate also suggests that the annihilation observed is controlled by threedimensional exciton diffusion. In the case of a preferentially onedimensional diffusion we would have strong time-dependence of $\gamma_{;}{ }^{11}$ hence, our data show that exciton diffusion is not confined to a single stack of molecules. The rate constant of annihilation which is controlled by three-dimensional exciton diffusion is

$$
\gamma=4 \pi D R_{a}
$$

where $D$ is exciton diffusivity and $R_{\mathrm{a}}$ is the annihilation radius, which is a distance at which annihilation is much faster than exciton diffusion away from the other exciton. Using the value of diffusivity $D=(1.6 \pm 0.3) \times 10^{-3} \mathrm{~cm}^{2} \mathrm{~s}^{-1}$ measured using surface quenching for as-cast DTS $\left(\mathrm{FBTTh}_{2}\right)_{2}$, we calculated an annihilation radius of $R_{\mathrm{a}}=1.1 \pm 0.4 \mathrm{~nm}$. Assuming that there is little change in $R_{\mathrm{a}}$ on annealing, we obtain a diffusivity, $D$, of $(3.2 \pm 0.3) \times 10^{-3} \mathrm{~cm}^{2} \mathrm{~s}^{-1}$ for annealed DTS $\left(\mathrm{FBTTh}_{2}\right)_{2}$. This result is consistent with the doubling of exciton diffusivity upon annealing, as previously observed with surface quenching. The consistent result from these two measurement techniques suggests that exciton diffusion is isotropic in DTS $\left(\mathrm{FBTTh}_{2}\right)_{2}$ films. The results are given in Table 1. The diffusion coefficient we obtain for annealed DTS $\left(\mathrm{FBTTh}_{2}\right)_{2}$ is higher than reported values for many other organic semiconductors, such as (1-9) $\times 10^{-4} \mathrm{~cm}^{2} \mathrm{~s}^{-1}$ measured for diketopyrrolopyrrole (DPP)-based small molecules, ${ }^{40,41}$ and $1 \times 10^{-4} \mathrm{~cm}^{2} \mathrm{~s}^{-1}$ for the efficient polymer donor PCDTBT. ${ }^{38}$ The diffusion coefficient measured for as-cast DTS $\left(\mathrm{FBTTh}_{2}\right)_{2}$ (before annealing) is comparable to that of $\mathrm{P} 3 \mathrm{HT}$, which is also a semicrystalline material. ${ }^{11,12,42}$

Exciton Diffusion Length. The length excitons diffuse in their lifetime in the operating conditions of solar cells can be estimated as the mean-square displacement using

$$
L_{Z D}=\sqrt{2 Z D \tau}
$$



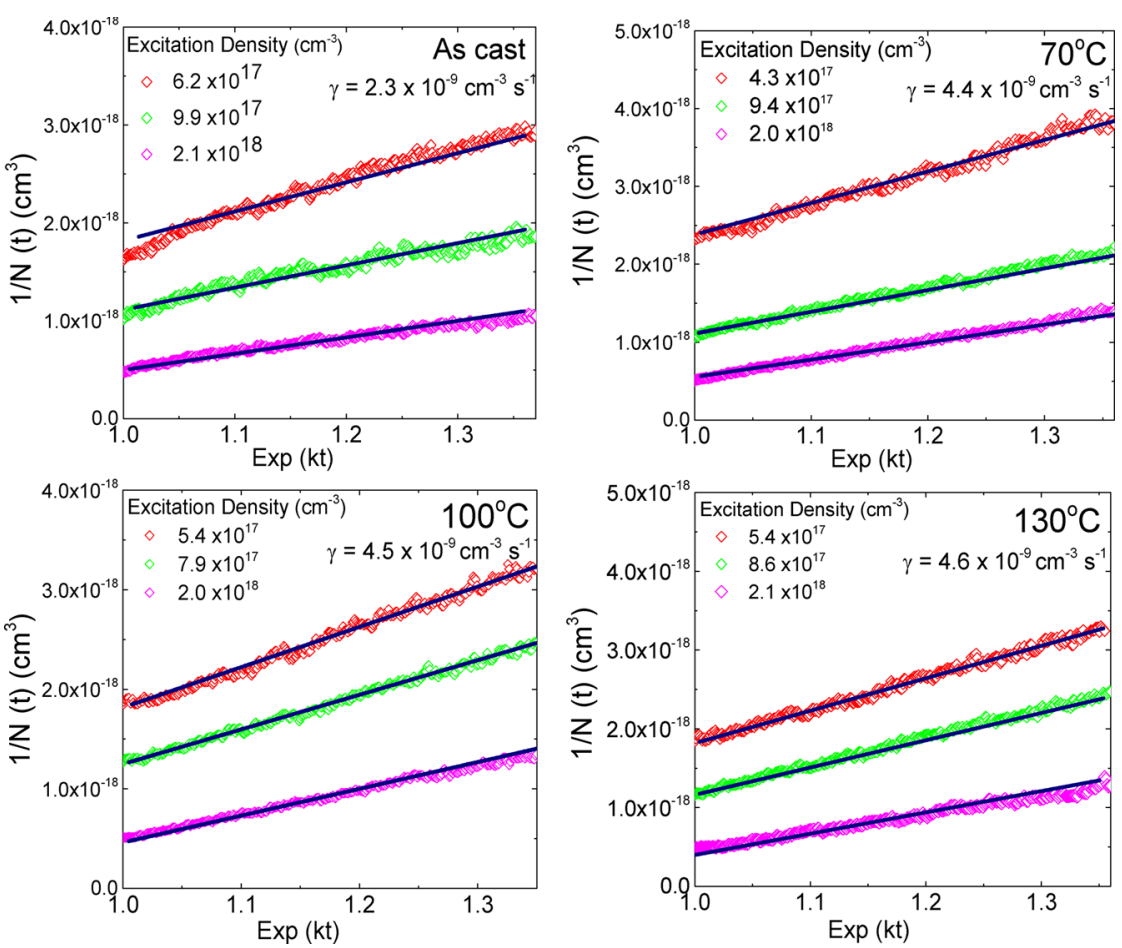

Figure 4. Reciprocal of exciton density vs $\exp (k t)$ in as-cast and annealed films and global linear fits to eq 6 with $\gamma$ values given in the insets.

Table 1. Rate Constant of Exciton-Exciton Annihilation $\gamma$, Exciton Diffusivity $D$, Exciton Lifetime $\tau$, and Two-Dimensional Diffusion Length

\begin{tabular}{|c|c|c|c|c|c|}
\hline annealing temp $\left({ }^{\circ} \mathrm{C}\right)$ & $D\left(\mathrm{~cm}^{2} \mathrm{~s}^{-1}\right)$ (from surface quenching) & $\gamma\left(\mathrm{cm}^{3} \mathrm{~s}^{-1}\right)$ & $D\left(\mathrm{~cm}^{2} \mathrm{~s}^{-1}\right)$ (from exciton annihilation) & $\tau(\mathrm{ps})$ & $L_{2 \mathrm{D}}(\mathrm{nm})$ \\
\hline as cast & $(1.6 \pm 0.3) \times 10^{-3}$ & $(2.3 \pm 0.1) \times 10^{-9}$ & $(1.6 \pm 0.3) \times 10^{-3}$ & 359 & $15 \pm 2$ \\
\hline 70 & & $(4.4 \pm 0.1) \times 10^{-9}$ & $(3.1 \pm 0.7) \times 10^{-3}$ & 380 & $22 \pm 3$ \\
\hline 100 & & $(4.5 \pm 0.1) \times 10^{-9}$ & $(3.1 \pm 0.7) \times 10^{-3}$ & 496 & $24 \pm 3$ \\
\hline 130 & $(3.6 \pm 0.7) \times 10^{-3}$ & $(4.6 \pm 0.1) \times 10^{-9}$ & $(3.2 \pm 0.7) \times 10^{-3}$ & 523 & $27 \pm 3$ \\
\hline
\end{tabular}

where $\tau$ is exciton lifetime measured at a very low excitation density (without annihilation) and $Z=1,2$, or 3 for one-, two-, or three-dimensional diffusion, respectively. As the donor molecules used in this study form elongated fibrous domains in photovoltaic blends with fullerenes, the excitons will find electron acceptors at the walls of these fibers; hence, only diffusion in the direction perpendicular to the fiber walls will result in charge generation. ${ }^{14}$ Therefore, exciton harvesting is determined by two-dimensional diffusion. The two-dimensional diffusion length $\left(L_{2 \mathrm{D}}\right)$ determined using eq 8 along with measured $\tau$ and $D$ values for as-cast and annealed films are given in Table 1 and shown in Figure 5. The highest $L_{2 \mathrm{D}}=27 \mathrm{~nm}$ is obtained for the film annealed at $130{ }^{\circ} \mathrm{C}$. It implies efficient exciton harvesting from large donor domains in optimized morphologies and shows the high tolerance of this material to the domain size for efficient charge generation. Our measured diffusion length is larger than in another study of this material using a different method (volume quenching) in which the quencher is dispersed in the film. ${ }^{32}$ We suspect that because of the polycrystalline nature of these films in such measurements the quencher is expelled from the crystalline domains when they are heated. ${ }^{43,44}$ Because excitons diffuse preferentially into these crystalline domains, their interaction with a dispersed quencher is weak, and quenching is slow. Therefore, although volume quenching measurements can provide information about the structure of the film, their ability to provide an accurate value of exciton diffusion length is hindered by the quencher being excluded from the crystalline domains.

The PL lifetimes measured in films were shorter than the $1.3 \mathrm{~ns}$ lifetime measured in solution. We attribute this to PL quenchers present in the film which excitons encounter while diffusing. The approximate density of these quenchers $N_{\mathrm{Q}}$ can be estimated using the Smoluchowski equation assuming that the intrinsic decay time of the exciton in film and solution is the same:

$$
\frac{1}{\tau}-\frac{1}{\tau_{\text {sol }}}=4 \pi D R_{\mathrm{Q}} N_{\mathrm{Q}}
$$

where $\tau$ and $\tau_{\text {sol }}$ are the PL lifetimes in film and solution, respectively, $D$ is exciton diffusivity, and $R_{Q}$ is the quenching radius. Assuming $R_{\mathrm{Q}}=1 \mathrm{~nm}$, we find that $N_{\mathrm{Q}}=1 \times 10^{18} \mathrm{~cm}^{-3}$ in the as-cast film and $N_{\mathrm{Q}}=3 \times 10^{17} \mathrm{~cm}^{-3}$ in the annealed film. We do not have information on the nature of these quenchers, but decreasing their density would increase exciton lifetime and hence the average diffusion length. In order to learn more about the effect of annealing, we measured fluorescence depolarization dynamics.

Fluorescence Depolarization. Figure 6 shows timeresolved fluorescence anisotropy obtained from the fluorescence kinetics measured with the excitation polarization set parallel and perpendicular to the detection polarization using eq 1 . This measurement tells us how quickly the polarization memory of excitation is lost by exciton diffusion. The initial anisotropy value of $\sim 0.3$ is very similar in both films, and faster depolarization 

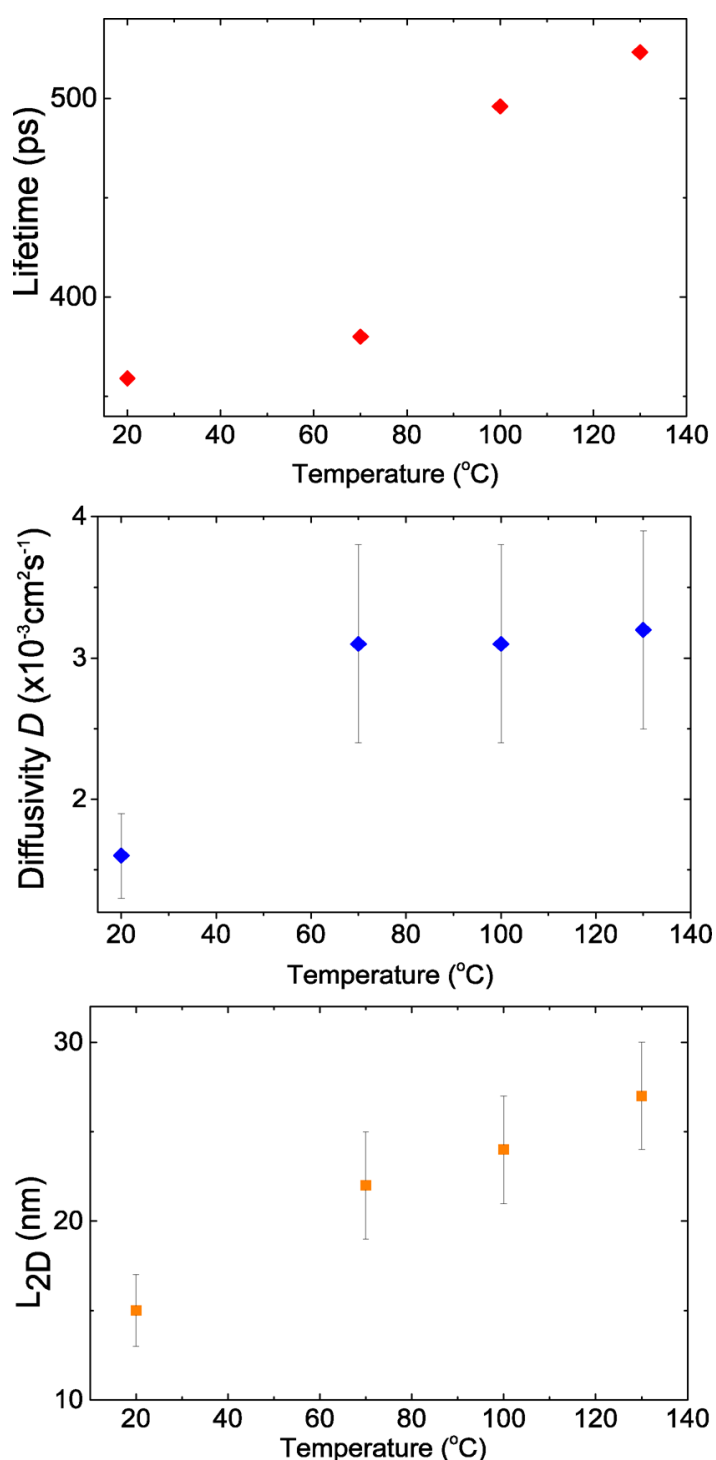

Figure 5. Exciton lifetime, diffusivity $(D)$, and two-dimensional exciton diffusion length $\left(L_{2 \mathrm{D}}\right)$ estimated using eq 7 for different annealing temperatures.

is observed in annealed film which is consistent with a higher average exciton diffusivity in this film. In the as-cast film, the fluorescence does not depolarize completely showing a value of $\sim 0.1$ at $1 \mathrm{~ns}$. With this value subtracted from $r(t)$ over the entire time range the depolarization dynamics of the as-cast film is identical to that of the annealed film. This suggests that some excitons get trapped very quickly in as-cast films and remain immobilized throughout their entire lifetime, so that they do not depolarize. Because the total anisotropy value is given by a sum of contributions from mobile and trapped excitons, the fraction of trapped excitons in the as-cast film can be estimated as a ratio of the long-time and initial anisotropy values, which points to about $30 \%$ of excitons becoming trapped in as-cast film. Then the diffusivity of mobile excitons in as-cast films is $\sim 30 \%$ higher than the ensemble-averaged diffusivity in this film measured by surface quenching and exciton-exciton annihilation which includes also the trapped excitons. This suggests that the diffusivity of mobile excitons in as-cast films is $22.1 \times 10^{-3} \mathrm{~cm}^{2} \mathrm{~s}^{-1}$, which is still lower than the diffusivity observed in annealed films. Despite slower diffusion, the mobile excitons in the as-cast film lose their

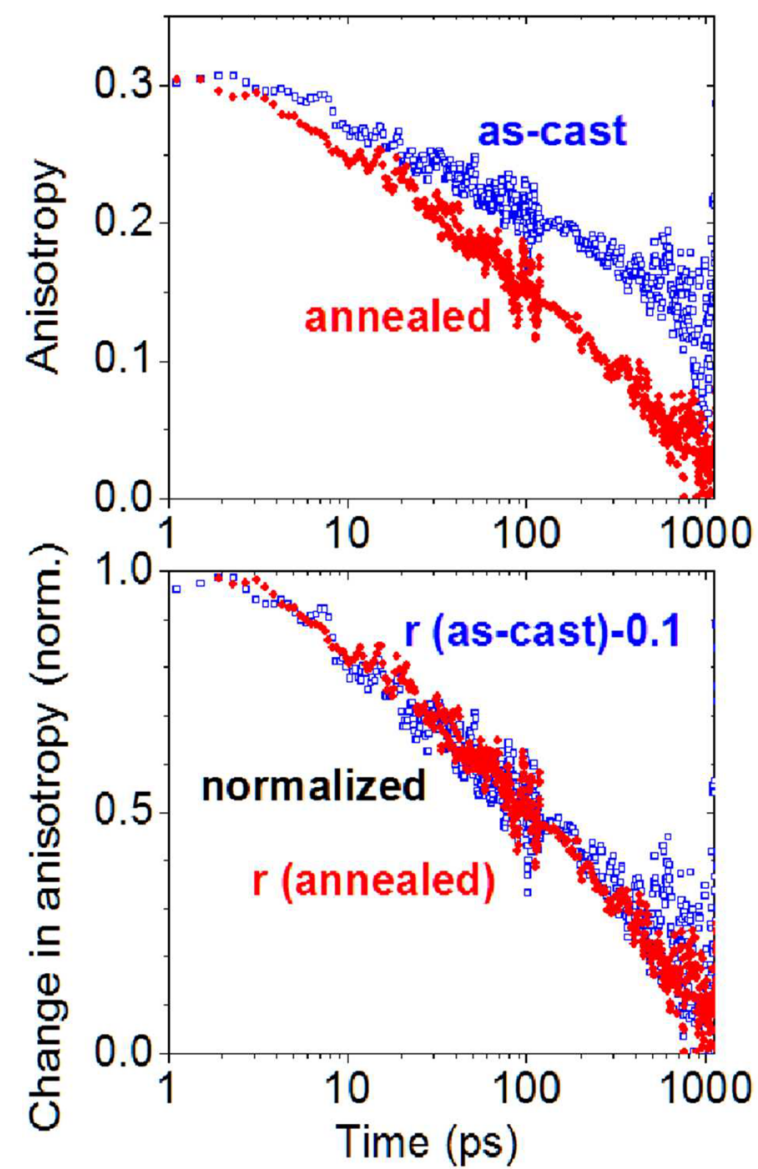

Figure 6. (top) Time-resolved fluorescence anisotropy measured in as-cast film of DTS $\left(\text { FBTTh }_{2}\right)_{2}$ and in the film annealed at $130{ }^{\circ} \mathrm{C}$ calculated from fluorescence kinetics using eq 1 . Fluorescence was detected in the spectral window of $750-850 \mathrm{~nm}$. In the bottom panel the value of 0.1 is subtracted from the as-cast anisotropy over the entire time range measured, and traces are normalized.

polarization memory at a similar rate to the faster excitons in the annealed film, which is consistent with the higher molecular order reported in annealed films. ${ }^{14}$ An alternative explanation for slower depolarization in the as-cast film could be the confinement of exciton diffusion to partially ordered molecular domains. The TEM imaging of as-cast films showed a homogeneous morphology, while fibrous structures appeared only after thermal annealing or when films were processed with solution additives. ${ }^{14,30}$ Hence, there are no obvious domains which could confine exciton diffusion in as-cast films. If such domains appeared in the bulk film, then we would expect much lower exciton diffusivity toward the substrate measured by surface quenching than that measured by exciton-exciton annihilation in the bulk of the film. The fact that both techniques give consistent exciton diffusivity suggests that exciton diffusion is predominantly isotropic while the residual anisotropy of fluorescence in as-cast films indicates trapping of some emissive excitons. We note that the excitons are still emissive in these traps, which distinguishes them from PL quenchers discussed above. Time-resolved measurements do not reveal the nature of the traps, but the red-shifted PL spectrum of the as-cast film (Figure 1) shows that some excitons have lower energy in this film as compared to annealed films. The lower energy sites can correspond to intermolecular aggregates in which the site energy is lowered by stronger Coulomb coupling between a few aggregated molecules. 


\section{CONCLUSIONS}

In examining exciton diffusion with different techniques, we showed that thermal annealing enhances the average exciton diffusivity and the mean diffusion length in DTS $\left(\mathrm{FBTTh}_{2}\right)_{2}$ films. Surface quenching of fluorescence and exciton-exciton annihilation show consistent results in that the average exciton diffusivity increases by a factor of 2 when the film is annealed. The exciton lifetime also increases upon annealing, and the two effects lead to an enhancement of the mean two-dimensional exciton diffusion length from 15 to $27 \mathrm{~nm}$. This long exciton diffusion length means that excitons can be harvested efficiently from a wide range of domain sizes. The fluorescence depolarization study shows that about $30 \%$ of excitons get trapped very quickly in as-cast films and remain immobilized throughout their entire lifetime and the rest of excitons diffuse slower as compared to annealed films. Overall, we conclude that thermal annealing leads to higher average diffusivity and longer exciton diffusion lengths in DTS $\left(\mathrm{FBTTh}_{2}\right)_{2}$ which allows excitons to diffuse further and provides efficient charge generation from large donor domains in bulk heterojunctions.

\section{ASSOCIATED CONTENT}

\section{S Supporting Information}

The Supporting Information is available free of charge on the ACS Publications website at DOI: 10.1021/acsami.6b16487.

Fluorescence spectra in solution and film, time-resolved fluorescence spectra in films, fluorescence decays in films on quenching substrate and in reference films, and fluorescence decays in thicker films (PDF)

\section{AUTHOR INFORMATION}

\section{Corresponding Author}

*E-mail: idws@st-andrews.ac.uk (I.D.W.S.).

ORCID $($ )

Mithun Chowdhury: 0000-0002-2513-6006

Ifor D. W. Samuel: 0000-0001-7821-7208

Notes

The authors declare no competing financial interest.

\section{ACKNOWLEDGMENTS}

We are grateful to the Engineering and Physical Sciences Research Council of the UK for financial support through grants EP/L505079/1, EP/L017008/1 and EP/J009016/1 and to The European Research Council of the European Union for support through grant 321305. I.D.W.S. acknowledges support from a Royal Society Wolfson Research Merit Award.

\section{REFERENCES}

(1) Xu, T.; Yu, L. How to Design Low Bandgap Polymers for Highly Efficient Organic Solar Cells. Mater. Today 2014, 17, 11-15.

(2) Li, G.; Zhu, R.; Yang, Y. Polymer Solar Cells. Nat. Photonics 2012, 6, $153-161$.

(3) Menke, S. M.; Holmes, R. J. Exciton Diffusion in Organic Photovoltaic Cells. Energy Environ. Sci. 2014, 7, 499-512.

(4) Lu, L.; Zheng, T.; Wu, Q.; Schneider, A. M.; Zhao, D.; Yu, L. Recent Advances in Bulk Heterojunction Polymer Solar Cells. Chem. Rev. 2015, 115, 12666-12731.

(5) Hedley, G. J.; Ruseckas, A.; Samuel, I. D. W. Light Harvesting for Organic Photovoltaics. Chem. Rev. 2017, 117, 796-837.

(6) Hoppe, H.; Sariciftci, N. S. Organic Solar Cells: An Overview. J. Mater. Res. 2004, 19, 1924-1945.
(7) Scharber, M. C.; Sariciftci, N. S. Efficiency of Bulk-Heterojunction Organic Solar Cells. Prog. Polym. Sci. 2013, 38, 1929-1940.

(8) Park, S. H.; Roy, A.; Beaupre, S.; Cho, S.; Coates, N.; Moon, J. S.; Moses, D.; Leclerc, M.; Lee, K.; Heeger, A. J. Bulk Heterojunction Solar Cells with Internal Quantum Efficiency Approaching 100\%. Nat. Photonics 2009, 3, 297-302.

(9) Li, W.; Hendriks, K. H.; Wienk, M. M.; Janssen, R. A. J. Diketopyrrolopyrrole Polymers for Organic Solar Cells. Acc. Chem. Res. 2016, 49, 78-85.

(10) Yu, G.; Gao, J.; Hummelen, J.; Wudl, F.; Heeger, A. J. Polymer Photovoltaic Cells: Enhanced Efficiencies via a Network of Internal Donor-Acceptor Heterojunctions. Science 1995, 270, 1789-1791.

(11) Masri, Z.; Ruseckas, A.; Emelianova, E. V.; Wang, L.; Bansal, A. K.; Matheson, A.; Lemke, H. T.; Nielsen, M. M.; Nguyen, H.; Coulembier, O.; Dubois, P.; Beljonne, D.; Samuel, I. D. W. Molecular Weight Dependence of Exciton Diffusion in Poly(3-hexylthiophene). Adv. Energy. Mater. 2013, 3, 1445-1453.

(12) Shaw, P. E.; Ruseckas, A.; Samuel, I. D. W. Exciton Diffusion Measurements in Poly(3-hexylthiophene). Adv. Mater. 2008, 20, 35163520.

(13) Mikhnenko, O. V.; Blom, P. W. M.; Nguyen, T.-Q. Exciton Diffusion in Organic Semiconductors. Energy Environ. Sci. 2015, 8, $1867-1888$

(14) Love, J. A.; Proctor, C. M.; Liu, J.; Takacs, C. J.; Sharenko, A.; van der Poll, T. S.; Heeger, A. J.; Bazan, G. C.; Nguyen, T.-Q. Film Morphology of High Efficiency Solution-Processed Small-Molecule Solar Cells. Adv. Funct. Mater. 2013, 23, 5019-5026.

(15) van der Poll, T. S.; Love, J. A.; Nguyen, T.-Q.; Bazan, G. C. NonBasic High-Performance Molecules for Solution-Processed Organic Solar Cells. Adv. Mater. 2012, 24, 3646-3649.

(16) Walker, B.; Kim, C.; Nguyen, T.-Q. Small Molecule SolutionProcessed Bulk Heterojunction Solar Cells. Chem. Mater. 2011, 23, 470-482.

(17) Takacs, C. J.; Collins, S. D.; Love, J. A.; Mikhailovsky, A. A.; Wynands, D.; Bazan, G. C.; Nguyen, T.-Q.; Heeger, A. J. Mapping Orientational Order in a Bulk Heterojunction Solar Cell with Polarization-Dependent Photoconductive Atomic Force Microscopy. ACS Nano 2014, 8, 8141-8151.

(18) Wan, X.; Liu, Y.; Wang, F.; Zhou, J.; Long, G.; Chen, Y. Improved Efficiency of Solution Processed Small Molecules Organic Solar Cells using Thermal Annealing. Org. Electron. 2013, 14, 1562-1569.

(19) Sun, Y.; Welch, G. C.; Leong, W. L.; Takacs, C. J.; Bazan, G. C.; Heeger, A. J. Solution-Processed Small-Molecule Solar Cells with 6.7\% Efficiency. Nat. Mater. 2011, 11, 44-48.

(20) Yi, Z.; Ni, W.; Zhang, Q.; Li, M.; Kan, B.; Wan, X.; Chen, Y. Effect of Thermal Annealing on Active Layer Morphology and Performance for Small Molecule Bulk Heterojunction Organic Solar Cells. J. Mater. Chem. C 2014, 2, 7247-7255.

(21) Leong, W. L.; Welch, G. C.; Kaake, L. G.; Takacs, C. J.; Sun, Y.; Bazan, G. C.; Heeger, A. J. Role of Trace Impurities in the Photovoltaic Performance of Solution Processed Small-Molecule Bulk Heterojunction Solar Cells. Chem. Sci. 2012, 3, 2103-2109.

(22) Kyaw, A. K. K.; Wang, D. H.; Gupta, V.; Leong, W. L.; Ke, L.; Bazan, G. C.; Heeger, A. J. Intensity Dependence of Current-Voltage Characteristics and Recombination in High-Efficiency SolutionProcessed Small-Molecule Solar Cells. ACS Nano 2013, 7, 4569-4577.

(23) Gupta, V.; Kyaw, A. K. K.; Wang, D. H.; Chand, S.; Bazan, G. C.; Heeger, A. J. Barium: An Efficient Cathode Layer for Bulkheterojunction Solar Cells. Sci. Rep. 2013, 3, 1965.

(24) Mishra, A.; Bäuerle, P. Small Molecule Organic Semiconductors on the Move: Promises for Future Solar Energy Technology. Angew. Chem., Int. Ed. 2012, 51, 2020-2067.

(25) Zhang, Q.; Kan, B.; Liu, F.; Long, G.; Wan, X.; Chen, X.; Zuo, Y.; Ni, W.; Zhang, H.; Li, M.; Hu, Z.; Huang, F.; Cao, Y.; Liang, Z.; Zhang, M.; Russell, T. P.; Chen, Y. Small-Molecule Solar Cells with Efficiency over 9\%. Nat. Photonics 2014, 9, 35-41.

(26) Zhao, Q.; Liu, J.; Wang, H.; Li, M.; Zhou, K.; Yang, H.; Han, Y. Balancing the $\mathrm{H}$ - and J-aggregation in DTS(PTTh2)2/PC70BM to 
yield a High Photovoltaic Efficiency. J. Mater. Chem. C 2015, 3, 81838192.

(27) Ko Kyaw, A. K.; Gehrig, D.; Zhang, J.; Huang, Y.; Bazan, G. C.; Laquai, F.; Nguyen, T.-Q. High Open-Circuit Voltage Small-Molecule p-DTS(FBTTh2)2:ICBA Bulk Heterojunction Solar Cells - Morphology, Excited-state Dynamics, and Photovoltaic Performance. J. Mater. Chem. A 2015, 3, 1530-1539.

(28) Herath, N.; Das, S.; Keum, J. K.; Zhu, J.; Kumar, R.; Ivanov, I. N.; Sumpter, B. G.; Browning, J. F.; Xiao, K.; Gu, G.; Joshi, P.; Smith, S.; Lauter, V. Peculiarity of Two Thermodynamically-Stable Morphologies and Their Impact on the Efficiency of Small Molecule Bulk Heterojunction Solar Cells. Sci. Rep. 2015, 5, 13407.

(29) Kan, B.; Li, M.; Zhang, Q.; Liu, F.; Wan, X.; Wang, Y.; Ni, W.; Long, G.; Yang, X.; Feng, H.; Zuo, Y.; Zhang, M.; Huang, F.; Cao, Y.; Russell, T. P.; Chen, Y. A Series of Simple Oligomer-like Small Molecules Based on Oligothiophenes for Solution-Processed Solar Cells with High Efficiency. J. Am. Chem. Soc. 2015, 137, 3886-3893.

(30) Huang, Y.; Wen, W.; Mukherjee, S.; Ade, H.; Kramer, E. J.; Bazan, G. C. High-Molecular-Weight Insulating Polymers Can Improve the Performance of Molecular Solar Cells. Adv. Mater. 2014, 26, 41684172.

(31) Kyaw, A. K. K.; Wang, D. H.; Gupta, V.; Zhang, J.; Chand, S.; Bazan, G. C.; Heeger, A. J. Efficient Solution-Processed Small-Molecule Solar Cells with Inverted Structure. Adv. Mater. 2013, 25, 2397-2402.

(32) Lin, J. D. A.; Mikhnenko, O. V.; van der Poll, T. S.; Bazan, G. C.; Nguyen, T.-Q. Temperature Dependence of Exciton Diffusion in a Small-Molecule Organic Semiconductor Processed With and Without Additive. Adv. Mater. 2015, 27, 2528-2532.

(33) Markov, D. E.; Amsterdam, E.; Blom, P. W. M.; Sieval, A. B.; Hummelen, J. C. Accurate Measurement of the Exciton Diffusion Length in a Conjugated Polymer Using a Heterostructure with a SideChain Cross-Linked Fullerene Layer. J. Phys. Chem. A 2005, 109, 52665274.

(34) Hsieh, C.-H.; Cheng, Y.-J.; Li, P.-J.; Chen, C.-H.; Dubosc, M.; Liang, R.-M.; Hsu, C.-S. Highly Efficient and Stable Inverted Polymer Solar Cells Integrated with a Cross-Linked Fullerene Material as an Interlayer. J. Am. Chem. Soc. 2010, 132, 4887-4893.

(35) Reichenberger, M.; Love, J. A.; Rudnick, A.; Bagnich, S.; Panzer, F.; Stradomska, A.; Bazan, G. C.; Nguyen, T.-Q.; Köhler, A. The Effect of Intermolecular Interaction on Excited States in p-DTS(FBTTH2)2. J. Chem. Phys. 2016, 144, 074904.

(36) Scully, S. R.; Armstrong, P. B.; Edder, C.; Fréchet, J. M. J.; McGehee, M. D. Long-Range Resonant Energy Transfer for Enhanced Exciton Harvesting for Organic Solar Cells. Adv. Mater. 2007, 19, 29612966.

(37) Scully, S. R.; McGehee, M. D. Effects of optical interference and energy transfer on exciton diffusion length measurements in organic semiconductors. J. Appl. Phys. 2006, 100, 034907.

(38) Ward, A. J.; Ruseckas, A.; Samuel, I. D. W. A Shift from Diffusion Assisted to Energy Transfer Controlled Fluorescence Quenching in Polymer-Fullerene Photovoltaic Blends. J. Phys. Chem. C 2012, 116, 23931-23937.

(39) Mikhnenko, O. V.; Cordella, F.; Sieval, A. B.; Hummelen, J. C.; Blom, P. W. M.; Loi, M. A. Exciton Quenching Close to PolymerVacuum Interface of Spin-Coated Films of Poly(p-phenylenevinylene) Derivative. J. Phys. Chem. B 2009, 113, 9104-9109.

(40) Lin, J. D. A.; Mikhnenko, O. V.; Chen, J.; Masri, Z.; Ruseckas, A.; Mikhailovsky, A.; Raab, R. P.; Liu, J.; Blom, P. W. M.; Loi, M. A.; GarciaCervera, C. J.; Samuel, I. D. W.; Nguyen, T.-Q. Systematic Study of Exciton Diffusion length in Organic Semiconductors by Six Experimental Methods. Mater. Horiz. 2014, 1, 280-285.

(41) Mikhnenko, O. V.; Lin, J.; Shu, Y.; Anthony, J. E.; Blom, P. W. M.; Nguyen, T.-Q.; Loi, M. A. Effect of Thermal Annealing on Exciton Diffusion in a Diketopyrrolopyrrole Derivative. Phys. Chem. Chem. Phys. 2012, 14, 14196-14201.

(42) Tamai, Y.; Ohkita, H.; Benten, H.; Ito, S. Exciton Diffusion in Conjugated Polymers: From Fundamental Understanding to Improvement in Photovoltaic Conversion Efficiency. J. Phys. Chem. Lett. 2015, 6, $3417-3428$.
(43) Brabec, C. J.; Heeney, M.; McCulloch, I.; Nelson, J. Influence of Blend Microstructure on Bulk Heterojunction Organic Photovoltaic Performance. Chem. Soc. Rev. 2011, 40, 1185-1199.

(44) Botiz, I.; Stingelin, N. Influence of Molecular Conformations and Microstructure on the Optoelectronic Properties of Conjugated Polymers. Materials 2014, 7, 2273-2300. 